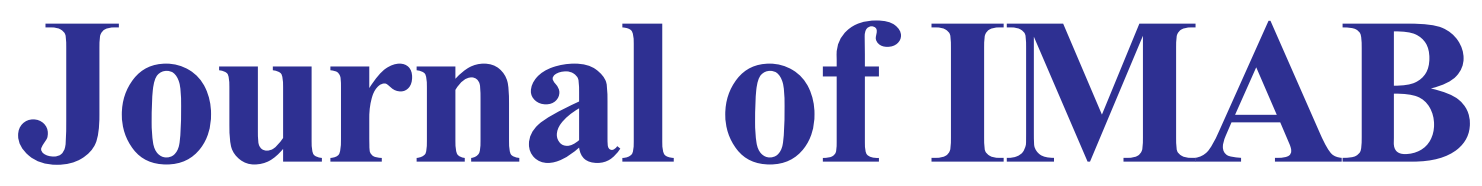

\title{
Annual Proceeding
} (Scientific Papers) 2012

\section{vol. 18, book 4 (July-December) part Oral\&Dental Medicine}

ISSN: 1312 773X (Online)

J of IMAB. 2012, 18(4) (July-December) DOI: $10.5272 /$ jimab.2012184
Publisher:

Peytchinski, Gospodin Iliev

Pleven, Bulgaria 


\title{
Journal of IMAB - Annual Proceeding (Scientific Papers)
}

\author{
Editor-in-chief: \\ Prof. Dr. Krassimir Metodiev \\ Deputy Editor-in-chief: \\ Assoc. prof. Dr. George Kyuchukov \\ Editors:
}

Prof. Dr. Angelina Kisselova-Yaneva (Responsible for part Oral\&Dental Medicine) Assoc. prof. Dr. Liana Gercheva (Responsible for part Medicine)

\section{Associate Editors:}

Prof. Dr. Bogdan Petrunov,

Prof. Dr. Bogomila Manevska,

Prof. Dr. Bojidar Popov,

Dora Peychinska,

Assoc. prof. Dr. Ivan Galabov,

Assoc. prof. Dr. Ivan Zlatarov,

Dr. Lyubomir Tzvetanov,

Paula Lazarova,

Assoc. prof. Dr. Pencho Kossev;

International Members:

Prof. Dr. Kurt G. Naber - Germany;

Prof. Dr. Mario Di Gioacchino - Italy;

Prof. Dr. Pio Conty - Italy;

Prof. Dr. Dieter Falkenhagen - Austria;

Prof. Dr. Ian Gould - UK;

Prof. Dr. Bulent Berkarda - Turkey;

Prof. Dr. Roman Kozlov - Russia;

Prof. Dr. Dan Engelhard - Israel;

Prof. Dr. Raphael Saginur - Canada;

\section{Part Medicine:}

Prof. Dr. Anelia Klissarova;

Assoc. prof. Dr. Dobrin Radev;

Assoc. prof. Dr. Ekaterina Softova;

Prof. Dr. Ekaterina Titianova;

Prof. Dr. Goce Spasovski - Macedonia;

Prof. Dr. Iskren Kotzev;

Assoc. prof. Dr. Ivelina Yordanova; Assoc. prof. Dr. Snejana Tisheva; Assoc. prof. Dr. Sonja GenadievaStavric-Macedonia;

Assoc. prof. Dr. Stoyanka Popova;

Assoc. prof. Dr. Valentin Stoyanov;

Prof. Dr. Zaharyi Krastev;
Part Oral\&Dental Medicine:

Prof. Dr. Alexandru Petre - Romania;

Dr. Assya Krasteva-Panova;

Dr. Bogdan Calenic - Romania;

Assoc. prof. Dr. Donka Kirova;

Assoc. prof. Dr. Elka Popova;

Assoc. prof. Dr. Hristina Lalabonova;

Assoc. prof. Dr. Hristina Mihailova;

Dr. Ilyana Stoeva;

Dr. Maria Dencheva;

Assoc. prof. Dr. Metodi Abadjiev;

Prof. Dr. Milena Peneva;

Dr. Nikolai Yanev;

Dr. Pavel Stanimirov;

Address for correspondence:

Journal of IMAB - Editor-in-chief

IMAB Headquarters in Medical University, Varna

55, M. Drinov str., 9002 Varna, Bulgaria,

Tel./Fax: +359/52/634 107, 379777

E-mail:kr.metod@vega.bg; kyuchger@abv.bg

\section{Publisher - Journal of IMAB:}

Peytchinski, Gospodin Iliev

complex Droujba bl. 116, ap. 41; 5806 Pleven, Bulgaria

E-mail: publisher_imab@abv.bg; publisher.imab@gmail.com;

\section{http://www.journal-imab-bg.org/}

Journal of IMAB - Annual Proceeding (Scientific Papers)

2012, vol. 18, book 4 (July-December) part Oral\&Dental Medicine

ISSN: 1312 773X (Online); DOI: 10.5272/jimab.2012184

Executive Director:

Gospodin Peytchinski 